\title{
FINGER TEXTURE VERIFICATION SYSTEMS BASED ON MULTIPLE SPECTRUM LIGHTING SENSORS WITH FOUR FUSION LEVELS
}

\author{
Musab T. Al-Kaltakchi ${ }^{1}$, Raid R. Omar ${ }^{2}$, Hikmat N. Abdullah ${ }^{3}$, Tingting Han ${ }^{4}$, Jonathon A. Chambers \\ ${ }^{1}$ College of Engineering, Al-Mustansiriya University, Baghdad, Iraq \\ 2 Technical Engineering College of Mosul, Northern Technical University, Iraq \\ ${ }^{3}$ College of Information Engineering, Al-Nahrain University, Baghdad, Iraq \\ ${ }^{4}$ Department of Computer Science and Information Systems, Birkbeck, University of London,London, UK \\ ${ }^{5}$ Department of Engineering, University of Leicester, Leicester, UK \\ musab.tahseen@gmail.com ${ }^{1}$, raidrafi1@gmail.com ${ }^{2}$, dr.h.abdullah@ieee.org ${ }^{3}$, tingting@dcs.bbk.ac.uk ${ }^{4}$, \\ Jonathon.Chambers@le.ac.uk ${ }^{5}$ \\ Received:29/8/2018, Accepted:28/9/2018
}

\begin{abstract}
Finger Texture (FT) is one of the most recent attractive biometric characteristic. It refers to a finger skin area which is restricted between the fingerprint and the palm print (just after including the lower knuckle). Different specifications for the FT can be obtained by employing multiple images spectrum of lights. Individual verification systems are established in this paper by using multiple spectrum FT specifications. The key idea here is that by combining two various spectrum lightings of FTs, high personal recognitions can be attained. Four types of fusion will be listed and explained here: Sensor Level Fusion (SLF), Feature Level Fusion (FLF), Score Level Fusion (ScLF) and Decision Level Fusion (DLF). Each fusion method is employed, examined for different rules and analysed. Then, the best performance procedure is benchmarked to be considered. From the database of Multiple Spectrum CASIA (MSCASIA), FT images have been collected. Two types of spectrum lights have been exploited (the wavelength of $460 \mathrm{~nm}$, which represents a Blue (BLU) light, and the White (WHT) light). Supporting comparisons were performed, including the state-of-the-art. Best recognition performance was recorded for the FLF based concatenation rule by improving the Equal Error Rate (EER) percentages from 5\% for the BLU and $7 \%$ for the WHT to $2 \%$
\end{abstract}

Keywords: Finger texture, Verification systems, Multiple spectrum, Fusion levels

\section{INTRODUCTION}

Biometric recognition refers to the applications that is used for verifying or identifying people. They have been widely discovered over the recent years by exploiting different biometric characteristics such as the earprint [1], palmprint [2], face [3] [4], sclera [5], iris [6][7], speaker [8,9,10,11], backhand patterns [12] and FT [13,14]. Other studies concentrated on more than one characteristic such as $[15,16,17,18]$. Any human finger has many characteristics and they can be easily noticed. Basically, each single finger includes one of the most well known biometric (the fingerprint). Furthermore, it contains other observable characteristics: the Finger Inner Knuckle (FIK), Finger Outer Knuckle (FOK), Finger Veins (FV), Finger Geometry (FG) and FT. The inner surface texture of fingers have recently been investigated. Fundamentally, they have similar features of the palmprints (the wrinkles and principal lines). The main features of the FT can be described by the visible vertical and horizontal lines, where these lines are clearly appeared. The FTs are unique, reliable and they are different even between the identical twins [19]. Many benefits can be gained by utilizing the FTs such as their features are stable, they do not affect by emotional feelings and they have rich patterns [19]. They exist on the palm side skin of the little finger, ring finger, middle finger and index finger. Fig.1 demonstrates the essential FT positions of the four fingers. 


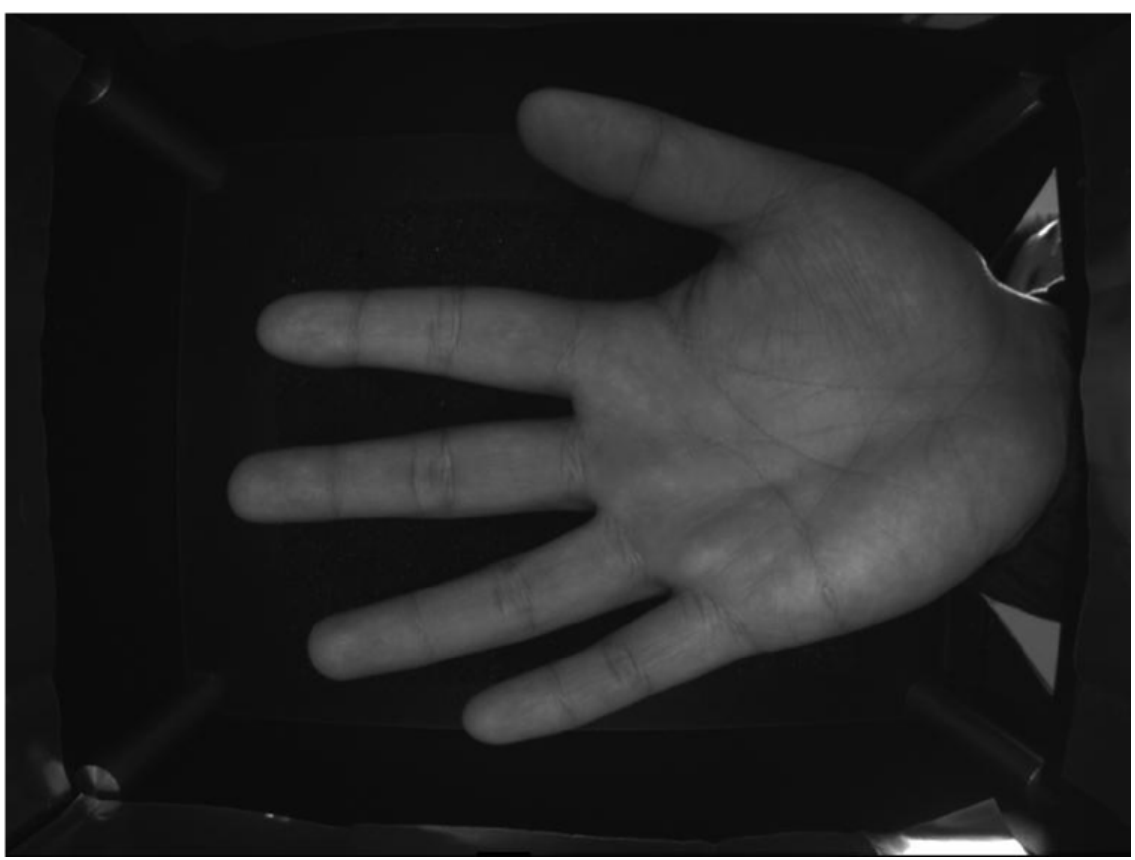

Figure 1: The essential locations of the FTs, they exist on the palm side skin of a little finger, ring finger, middle finger and index finger[20]

Fusion strategies have been employed in different fields and applications as in $[8,21]$, where various fusions between different features were used in order to improve the recognition performance. The major advantage of utilizing fusions in a biometric system is to decrease the error rate. Fig.2 shows fusion levels and rules and how they have been applied in this paper. The aim and contribution here are to investigate a high performance individual verification system by combining multiple spectrum FT specifications. Various fusion levels and rules are applied, studied and evaluated in different verification systems. Then, the procedure which attaining the best result is highlighted and considered. The organization of this paper after the introduction can be described as follows: the literature review will be reviewed in Section 2, the methodology of The suggested verification framework will be illustrated in Section 3, the four fusion levels and rules will be explained in Section 4, the achieved outcomes will be clarified in Section 5 and the paper will be concluded in Section 6.

\section{Literature Review}

Several studies have investigated the FT for personal recognition. In 2005, Ribaric and Fratric [22] suggested the first approach of FTs to be combined with palm print. The eigen values of the finger and palm were employed as types of feature extraction. In 2009, Pavesic et al.[23] proposed a human authentication system based on the fusion between the FTs and fingerprints of four fingers. In this study, the authors provide comparisons of three feature extraction methods. These are the Regularised - Direct Linear Discriminant Analysis (RDLDA), Most Discriminant Features (MDF) and Principal Component Analysis (PCA). Then, they reported that the RDLDA feature extraction method obtained the best results. In 2010, Michael et al.[24] presented a recognition fusion system based on the palm print and FTs. A tracking procedure 


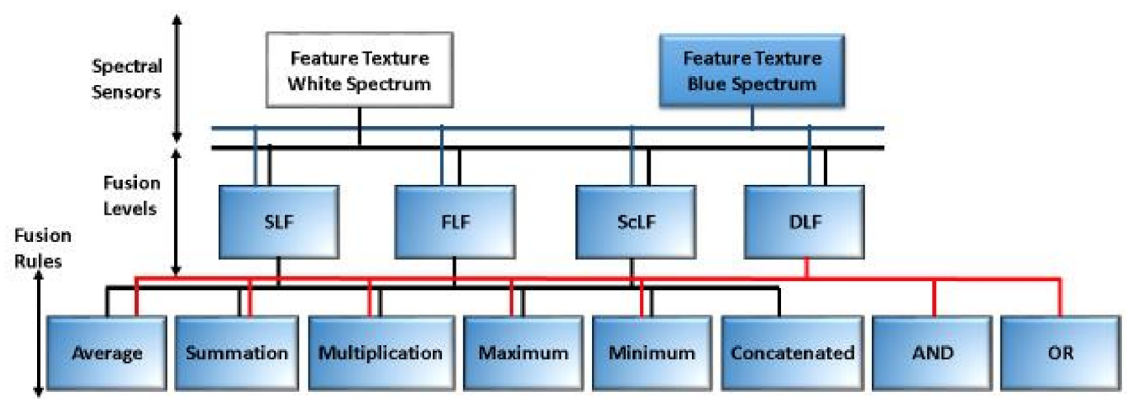

Figure 2: Different fusion levels and rules between the two spectra of FTs

named a projectile was proposed to detect the locations of the five fingers from a video stream. The ridgelet transform was applied as a feature extraction for the FTs. On the other hand, a directional coding technique was presented to extract the features of the palm print. In 2010, Michael et al. [25] described the same approaches as in [24] but with a difference of employing only four fingers and ignoring the thumb. In 2011, Kanhangad et al. [26] explained a unified framework for the case of verification. Various biometric characteristics of the hand were been investigated in this work, such as FTs, palm print, hand geometry, 3D palm print and 3D hand geometry. Each one of these characteristics was separately examined and then various fusions between the determined characteristics were performed. The feature extraction method which was used to analyse the FT patterns was the Competitive Coding (CompCode)[27] . In 2012, Zhang et al. [28] proposed a score fusion method using the summation rule. The fusion was performed between two types of biometrics, the palm print and the FT of a middle finger only. The image of the segmented middle finger was treated as a FT region. A two dimensional wavelet technique was employed to collect the features of both biometrics. The wavelet coefficients of approximation, and horizontal and vertical details were collected separately for each biometric. An average filter was applied to the palm print coefficients of the horizontal and vertical details. Consequently, the feature fusion was implemented between all coefficients of each biometric. Then, the score fusion was executed after the matching operations. In 2015, Al-Nima et al. [29] suggested extracting all the FT parts of the four fingers. In this work, it was cited that collecting more FT patterns will lead to increase the recognition performance. Furthermore, a new feature extraction method named the Image Feature Enhancement (IFE) was adopted. In 2016, Al-Nima et al. [30] suggested a novel approach to establish the Receiver Operating Characteristic (ROC) graph from an Artificial Probabilistic Neural Network (APNN) by employing the FT characteristic. Three feature extraction methods were examined: the Local Binary Pattern (LBP) with a statistical calculation method called the Coefficient of Variance (CV), the Gabor filter with the CV and the statistical CV computations alone in order to present the accuracy of the suggested approach. The best performance was recorded for the LBP with the CV. Finally, in 2016, AlNima et al. [31] proposed three main contributions: a robust finger segmentation approach, an enhanced feature extraction method and a novel scheme to salvage the missing FT information. Such work has drawn considerable state-of-the-art attention in the case of FT recognition. This study has employed three databases to support the results of the suggested approaches, one of which was the MSCASIA database, specifically with spectrum $460 \mathrm{~nm}$ 
images. It has been proved that by employing various spectrum lightings at the capturing step, different FT specifications can be clarified. This inspired the approach of this study. It can be observed from the previous literature that utilizing FTs for biometric recognition has always been considered as a part of study, except in [29,31]. Also, no publication has concentrated on combining various FT specifications that captured by using different spectra. In addition, the MSCASIA database has been found to be beneficial to support this work. This is because each participant has provided six types of images, and each was captured with a certain spectrum lighting. Therefore, by utilizing the hand images that contain various FT specifications, an effective human biometric system can be constructed

\section{Finger Texture Verification Framework}

A verification framework based on the FT biometric is suggested. It consists of two stages (learning and testing) with multiple processes. The flowchart of the suggested framework is given in Fig.3. The processes are started by the learning stage as structured below:

(1) Acquiring a hand image.

(2) Consequently, segmenting the fingers can be performed according to [31] or [13] .

(3) The Region of Interest (ROI) can be determined for the images of four fingers (index, middle, ring and little) by applying the largest adaptive rectangle method [26] . Each ROI is normalized to a size of $30 \times 150$ pixels as this size is confirmed by many papers such as [13], [20], [31], [32] .

(4) Afterwords, a feature extraction method is applied to the ROI images by utilizing the Enhanced Local Line Binary Pattern (ELLBP) and the CV computations as in [31]. The reason of choosing the ELLBP is that this method can professionally analyse the main FT features (the horizontal and vertical lines) as confirmed in [31]. The essential ELLBP calculations are given in the following equations:

$$
\begin{aligned}
V E C_{1}(L, c)= & \sum_{n=1}^{c-1} f\left(k h_{n}-k h_{c}\right) 2^{(c-n-1)}+\sum_{n=c+1}^{L} f\left(k h_{n}-k h_{c}\right) 2^{(n-c-1)} \\
V E C_{2}(L, c)= & \sum_{n=1}^{c-1} f\left(k v_{n}-k v_{c}\right) 2^{(c-n-1)}+\sum_{n=c+1}^{L} f\left(k v_{n}-k v_{c}\right) 2^{(n-c-1)} \\
& E L L B P_{a v}=\left(v_{1} \times V E C_{1}\right)+\left(v_{2} \times V E C_{2}\right)
\end{aligned}
$$

Where:

$$
f(x)= \begin{cases}1, & x \geq 0 \\ 0, & x<0\end{cases}
$$

$V E C_{1}$ represents the computed horizontal value, $V E C_{2}$ represents the computed vertical value, $L$ represents the length of the vertical and horizontal vectors, $c$ is a centre pixel location, $k h_{c}$ represents the centre of a horizontal vector, $k v_{c}$ represents the centre of a vertical vector, $k h_{n}$ represents the neighbourhoods pixels of the horizontal vector, $k v_{n}$ represents the neighbourhoods pixels of the vertical vector, and $v_{1}$ and $v_{2}$ are the weighted summation variables $\left(v_{1}+v_{2}=1\right)[31]$. 


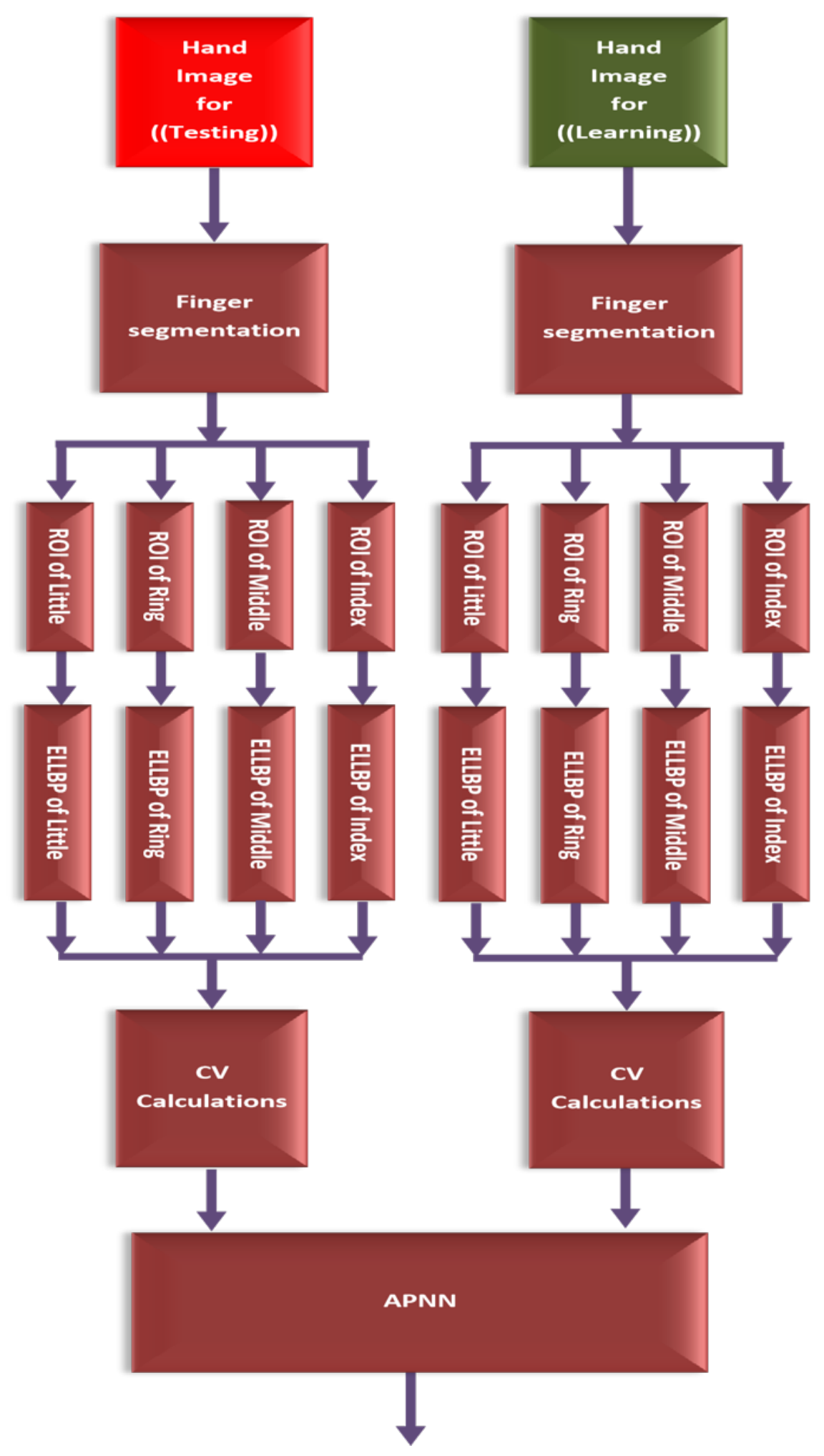

Figure 3: The flowchart of the suggested framework 
(5) It has been cited that using CV calculations can provide effective descriptions for the variances of the featured images. The resulting images have been partitioned into non-overlapping windows with a fixed size of $5 \times 5$ and the CV value is computed for each window [20], [29], [30], [33]. To calculate the CV values, the following equations have been exploited [34]:

$$
\begin{gathered}
A V_{\text {seg }}=\frac{1}{m} \sum_{i=1}^{m} w_{i n} \\
S D_{\text {win }}=\sqrt{\frac{1}{m-1} \sum_{i=1}^{m}\left(\operatorname{seg}_{i}-A V_{\text {win }}\right)^{2}} \\
C V_{\text {win }}=\frac{S D_{\text {win }}}{A V_{w i n}}
\end{gathered}
$$

Where $m$ represents the number of considered pixels, win represents the selected ( $5 \times 5$ pixels) window, $A V$ represents the average, $S D$ represents the standard deviation and $C V$ represents the coefficient of variance.

(6) Hereafter, an APNN is applied in terms of verification. The architecture of the APNN is given in Fig. 4. Principally, the calculations of the APNN are as follows:

After constructing the input values, hidden or pattern layer values are extracted according to [35], [36] :

$$
P_{u, v}=\exp \left[-\frac{\left(r-g_{u, v}\right)^{T}\left(r-g_{u, v}\right)}{2 \beta^{2}}\right], \quad u=1,2, \ldots, z \quad, \quad v \quad=\quad 1,2, \ldots, s
$$

Where $P_{u, v}$ is the value of a hidden or pattern neuron, $\mathbf{r}$ is the vector of input values, $\mathbf{g}_{u, v}$ represents the weights vector, $\beta$ represents the Gaussian function smoothing parameter, $z$ represents the number of learning patterns for each subject or class and $s$ represents the number of classes.

The summation layer then receives the values of the pattern layer and specifies each input vector to its assigned class as:

$$
A_{v}=\frac{1}{z} \sum_{i=1}^{z} P_{u, v}, \quad j=1,2, \ldots, s
$$

Where $A_{v}$ represents the value of a summation neuron.

The decision layer uses Equation (10) to perform the winner takes all rule:

$$
Q_{v}=\left\{\begin{array}{cc}
1 & \text { if } A_{v}=\max \\
0 & \text { otherwise }
\end{array}, \quad v=1,2, \ldots, s\right.
$$

Where $Q_{v}$ represents the value of a decision neuron and $\max$ represents the maximum value.

During the learning stage, the weights are established by the APNN. These weights are later exploited for the testing. New input samples are used in the testing stage and the same learning processes are followed in order to evaluate the verification performance. Significant advantages can be observed for the APNN [37]: it measures the confidence (probability) of any input before producing the output; flexible to add new participants or delete existed participants; the learning time is very short because it requires only one epoch to perform the learning stage; and it is not influenced by the local minimum. 


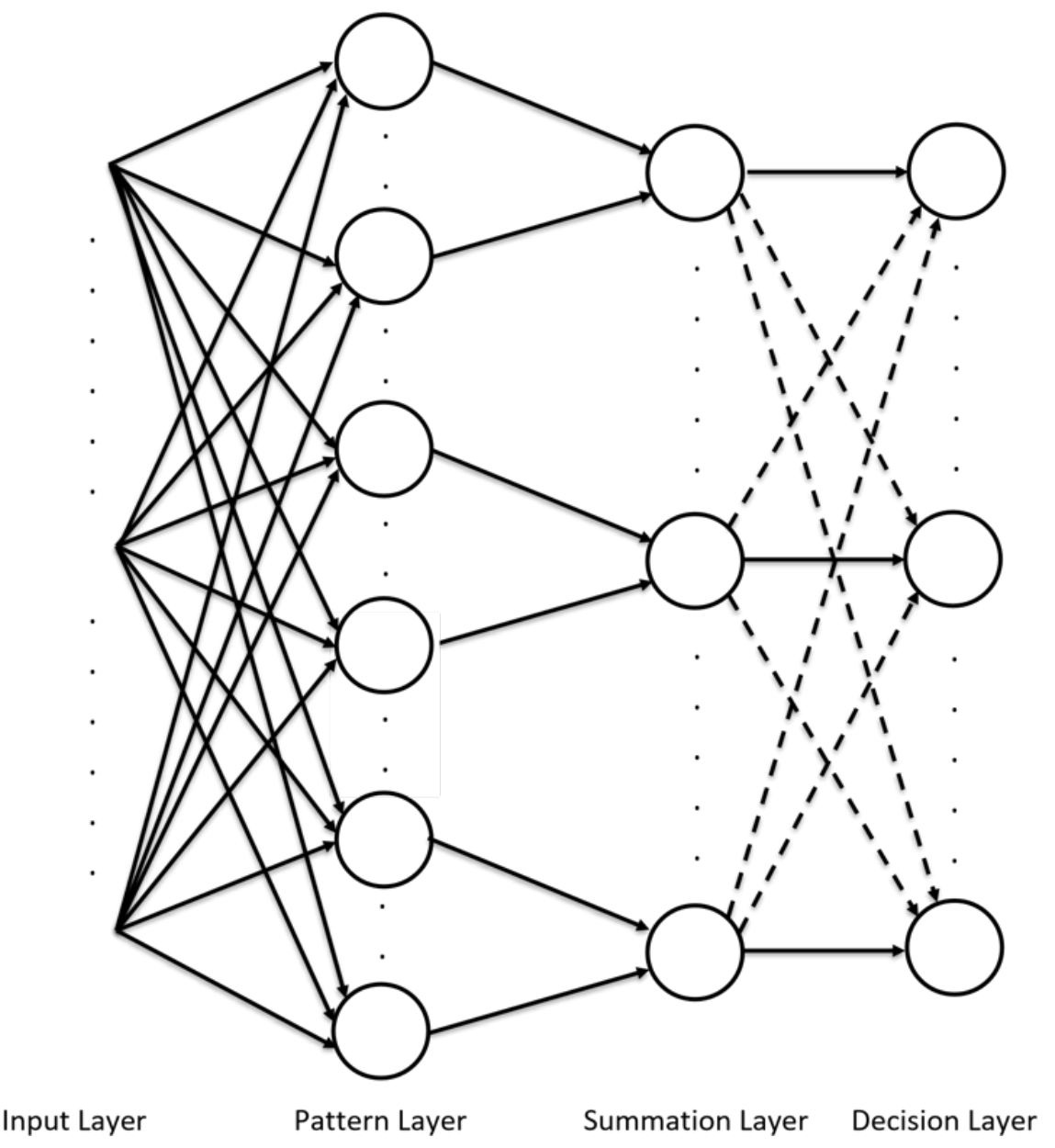

Figure 4: The main structure of the APNN, it includes input, pattern, summation and decision layers

Applying the FTs to the APNN is similar to the one that suggested in [32] and this confirms the proficiency of our proposed verification method.

\section{BIOMETRIC Fusion TyPES}

1) Fusion Levels: Fusions are usually employed to improve the performance of biometric systems. Four fusion levels are categorized as follows: SLF, FLF, ScLF and DLF [38] . They can be illustrated as follows:

(1) Sensor Level Fusion (SLF): this fusion is constructed at the sensor level. In this type, multiple sensors must be used in the biometric system. The utilized sensors can provide different specifications for the same input. By fusing the collected information, more data will be considered in the system.

(2) Feature Level Fusion (FLF): this fusion is used in the feature extraction step. The same inputs are treated with various feature extraction methods. So, the resulting data is fused together and more feature extractions can be analysed by the system. A reduction process may be required in this fusion level. 
(3) Score Level Fusion (ScLF): this fusion considers the score combinations of multiple matchers. The key idea here is that different scores can be generated from different matchers. So, by combining them the biometric system outputs can be influenced.

(4) Decision Level Fusion (DLF): this fusion usually performs at the last step in any biometric system. Different recognition decisions can be combined here. Therefore, the biometric system outcomes may be affected.

In this paper, the SLF was applied for the captured finger images before the starting operations, as in this case the input information considers the fusion between the FT images of the two employed spectra. The FLF was applied for the completed extracted features, represented by the CVs, before the APNN. For the ScLF, the outputs of the summation layer are considered to be the scores of the APNN, because the summation layer holds the real output values according to [30] . For the DLF, two full systems for the finger images of the two determined spectrum lightings are established and then the final decision of the two APNNs are competed to perform the employed fusion rules.

2) Fusion Rules: There are various statistical rules which can be employed to perform a fusion operation. Some of them are known, for example maximum, minimum, AND and OR rules. Others are designed as suggested rules according to certain applications [39] . In this paper, the following fusion rules have been considered: average, summation, multiplication, maximum, minimum, concatenation, AND and OR.

First of all, the average fusion rule between two vectors is represented by the mean combinations of their components [8], [21] . It can be described in Equation (11) as follows:

$$
A v e_{k}=\frac{f_{k}+b_{k}}{2}
$$

Where: Ave is the resulting average vector, $k$ is the counter of the elements in each vector $(k=1,2, \ldots, q), q$ is the total number of elements in each vector and it has to be equal for all vectors, and $\mathbf{f}$ and $\mathbf{b}$ are the first and second vectors to be fused.

The summation fusion rule is almost similar to the average fusion rule except the dividing part. Thus, it can be derived from the previous equation as follows:

$$
\operatorname{Sum}_{k}=f_{k}+b_{k}
$$

Where: Sum is the resulting summation vector.

Similarly, the multiplication rule can be represented as follows:

$$
M u l_{k}=f_{k} \cdot b_{k}
$$

Where: Mul is the resulting multiplication vector.

The maximum fusion rule between two vectors applies by selecting the maximum values between their components [8], [21]. It can be illustrated as follows:

$$
\operatorname{Max}_{k}=\operatorname{MAX}\left(f_{k}, b_{k}\right)
$$

Where: Max is the resulting maximum vector which contains the selected maximum elements of the two given vectors and MAX is the maximum operation. 
Likewise, the minimum fusion rule can be given as follows:

$$
\operatorname{Min}_{k}=\operatorname{MIN}\left(f_{k}, b_{k}\right)
$$

Where: Min is the resulting minimum vector which contains the selected minimum elements of the two given vectors and MIN is the minimum operation.

The concatenation fusion rule between two vectors associates the elements of these vectors into one vector. It can be highlighted as follows:

$$
\operatorname{Con}_{k}=\left[f_{k}, b_{k}\right]
$$

Where: Con is the resulting concatenation vector which compromises the associated elements of both fused vectors.

It is not feasible to perform the concatenation rule in the DLF due to the fact that the final output decision needs to be collected after this level and implementing the concatenation rule here will not determine the last pursuing outputs. Instead, certain fusion rules that are commonly used are examined. These are the AND and OR fusion rules, as these rules require logical information to be applied [40] . So, they can be employed in the DLF. The main equations of these rules are given in (17) and (18):

$$
\begin{aligned}
A n d_{k} & =f_{k} A N D b_{k} \\
O r_{k} & =f_{k} O R b_{k}
\end{aligned}
$$

Where: And and Or represent the resulting logical operations vectors, AND represents the logical AND operation and OR represents the logical OR operation.

\section{Results AND Discussion}

1) CASIA Multiple Spectrum Palmprint Image Database (Version 1.0): The CASIA Multiple Spectrum Palmprint Image Database (Version 1.0) [41] is found to be useful in this study, for simplicity the name of this database is abbreviated to MSCASIA. Multiple spectrum light sensors were utilized in the MSCASIA database to acquire various hand specifications. Fundamentally, when different spectra of lights are supplied to a hand skin different features can be revealed. This is because that each spectrum can penetrate to different depth through the skin. Six types of hand images were acquired by using special multiple spectrum device. This device was designed to capture contactless or touchless hand images, so, there were no restrictions to a specific position. A dark environment and lightings, which are equally sourced from the bottom of the device, were constructed. A camera was positioned underneath the acquisition environment. The users were asked to open their fingers inside the designed environment. A total of one hundred participants were contributed. Six hand images were acquired in 2 sessions from each participant, so, 3 hand images were acquired in each session. The elapsed time between the sessions was over a month. The multiple spectra that were produced by the lights are as follows: WHT, 940 $\mathrm{nm}, 850 \mathrm{~nm}, 700 \mathrm{~nm}, 630 \mathrm{~nm}$ and $460 \mathrm{~nm}$. The overall acquired hand images is 7200, they are all of an extension JPEG and of a type grayscale. A low resolution of 576 X 768 pixels was assigned to each image [41] . Samples of six multiple spectrum hand images belonging to the same person or subject are shown in Fig.5. From the MSCASIA database, images of the wavelength $460 \mathrm{~nm}$ (or BLU spectrum) are chosen to be utilized, because this spectrum involves FTs as highlighted 
TABLE I

BETWEen VARIOUS TYPES OF THE LBP FOR THE FTS OF FOUR FINGERS

\begin{tabular}{|c|c|c|c|}
\hline \hline \multicolumn{5}{|c}{ Spectrum WHT of the MSCASIA database } \\
\hline Reference & Approach & Used variables & EER \\
\hline$[44]$ & CSLBP & Radius=1, Neighbourhoods $=8$ & $69 \%$ \\
\hline$[45]$ & CBP & Radius $=2$, Neighbourhoods $=8$ & $60 \%$ \\
\hline$[46]$ & LGC-HD & Radius $=1$, Neighbourhoods $=8$ & $22 \%$ \\
\hline$[47]$ & TPLBP & Threshold $=0.01$, Step jump $=5$, No. of patches $=8$, Radius $=2$ and Patch size $=3$ & $13 \%$ \\
\hline$[48]$ & SLBP & Radius $=1$, Neighbourhoods $=8$ & $12 \%$ \\
\hline Proposed as new work & ELLBP & Length of horizontal and vertical vectors $=17$ pixels & $7 \%$ \\
\hline \hline
\end{tabular}

\begin{tabular}{|c|c|c|c|}
\hline \multicolumn{5}{|c|}{ Spectrum BLU of the MSCASIA database } \\
\hline Reference & Approach & Used variables & EER \\
\hline$[44]$ & CSLBP & Radius $=1$, Neighbourhoods $=8$ & $74 \%$ \\
\hline$[45]$ & CBP & Radius $=2$, Neighbourhoods $=8$ & $78 \%$ \\
\hline$[46]$ & LGC-HD & Radius $=1$, Neighbourhoods $=8$ & $27 \%$ \\
\hline$[47]$ & TPLBP & Threshold $=0.01$, Step jump $=5$, No. of patches $=8$, Radius $=2$ and Patch size $=3$ & $34 \%$ \\
\hline$[48]$ & SLBP & Radius $=1$, Neighbourhoods $=8$ & $32 \%$ \\
\hline Proposed as in $[31]$ & ELLBP & Length of horizontal and vertical vectors $=17$ pixels & $5 \%$ \\
\hline
\end{tabular}

in [42], [43] . Furthermore, due to the fact that the FTs are always provided by the normal lighting, the images that were captured by applying the WHT light are selected. This can be considered as a good opportunity to work with different FT specifications. Four types of fingers (the little, ring, middle and index fingers) were extracted from the selected spectrum types of right hand images. Overall, 4800 images of fingers were exploited in this work. 2400 images of fingers belong to the WHT spectrum and 2400 images of fingers belong to the BLU spectrum. Firstly, 4000 images were used in the learning stage and 800 images were used in the testing stage as exploited in the state-of-the-art studies [20,29,30,31]. Secondly, we have changed the number of learning and testing samples (by decreasing the number of learning images and increasing the number of testing images) and we have recorded the obtained evaluations. The FTs of both spectra have been evaluated in the case of the feature extraction. Various types of modified LBP versions were examined. Table I shows comparisons of each employed spectrum with some enhanced LBP methods. It can be observed from this table that worst EER values have been recorded for the methods that considered the diagonal patterns and implement fewer calculations for all the essential pattern directions (vertical, horizontal and both diagonals), such as the Centralized Binary Patterns (CBP) and the Centre - Symmetric Local Binary Pattern (CS-LBP). The Local Gradient Coding - Horizontal and Diagonal (LGC-HD) approach has obtained high percentage values of the EER as it calculates the gradients of the diagonal features, but it considers useful computations for the gradient of the horizontal features. Nevertheless, it discards the computations of the vertical features, where these features can be considered as very significant FT specifications. Both the Simplified LBP (SLBP) and Three - Patch LBP (TPLBP) have reported equivalent percentages. For the FTs of the WHT spectrum, their values are low. On the other hand, for the FTs of the BLU spectrum, their values are high. This is due to their considerations of the clear features that could be acquired under the conventional lighting, however.

They show high percentages with the micro-textures that captured by applying the low spectrum wavelength. The ELLBP 

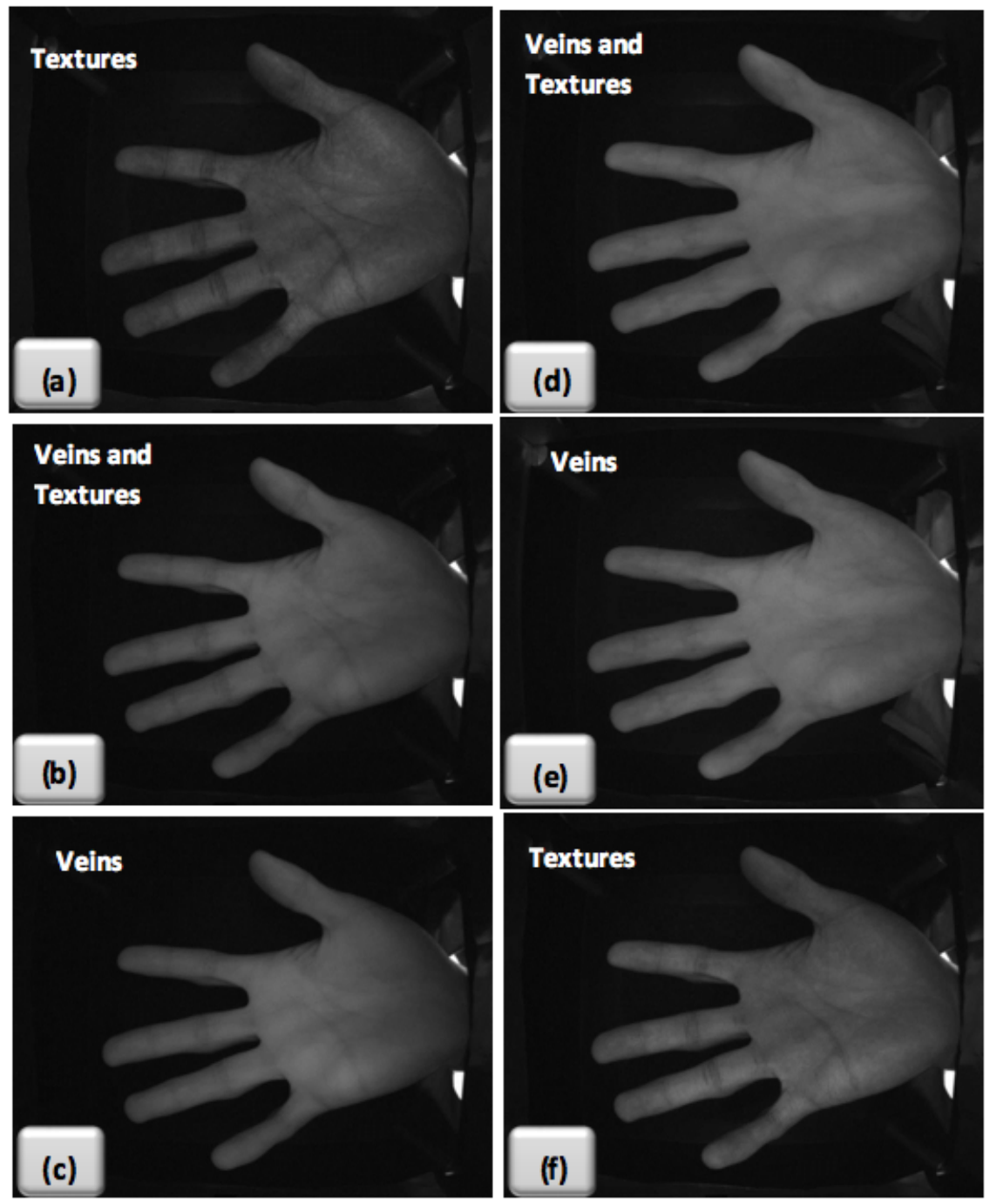

Figure 5: Samples of a right hand image from the MSCASIA database for certain wavelengths of lightings. (a) A wavelength of $460 \mathrm{~nm}$ contains only FTs without veins; (b) A wavelength of $630 \mathrm{~nm}$ contains FTs with veins; (c) A wavelength of $700 \mathrm{~nm}$ contains only veins; (d) A wavelength of $850 \mathrm{~nm}$ contains FTs with veins; (e) A wavelength of $940 \mathrm{~nm}$ contains only veins; and (f) Wavelengths of WHT contains only FTs [41]

method has benchmarked the lowest EER percentages here, so, this approach is able to provide effective analysis for the multiple spectrum features of the FT. With more explanation, the ELLBP concentrates on the essential features of FTs (the horizontal and vertical lines) and it well considers their weights that given in Equation (3). The best ELLBP weights for spectrum BLU have been cited as $v 1=0.2$ and v2 $=0.8$ [31]. Nevertheless, the best ELLBP weights for the WHT light have experimentally been found as $\mathrm{v} 1=0.7$ and $\mathrm{v} 2=0.3$. It has been observed that these values are perfectly equal to the weights of the IIT Delhi database [31], which was collected by using a normal lighting too. In the case of fusion, the four combination levels which have been examined are: the SLF, FLF, ScLF and DLF. Furthermore, the 
TABLE II

EER RESUlts FOR THE DiFFERENT Fusion METHOdS

\begin{tabular}{lllllllll}
\hline \hline Fusion level & & & & EER & & & \\
& Average & Summation & Multiplication & Maximum & Minimum & Concatenation & AND \\
& rule & rule & rule & rule & rule & rule & rule & rule \\
\hline SLF & $4 \%$ & $4 \%$ & $97 \%$ & $5 \%$ & $5 \%$ & $2 \%$ & - & - \\
FLF & $2 \%$ & $2 \%$ & $18 \%$ & $3 \%$ & $7 \%$ & $2 \%$ & - \\
ScLF & $5 \%$ & $5 \%$ & $2 \%$ & $5 \%$ & $4 \%$ & $12 \%$ & - \\
DLF & $5 \%$ & $5 \%$ & $11 \%$ & $5 \%$ & $11 \%$ & - & $11 \%$ & $5 \%$ \\
\hline
\end{tabular}

six well-known rules that have been investigated are: the average, summation, multiplication, maximum, minimum and concatenation rules. As mentioned, the concatenation rule can not be employed in the DLF because it can not provide a clear output decision suitable for the desired number of outputs. Thus, the concatenation is replaced in this level of fusion by the AND and OR rules, which are more suitable here because they are logical operations and the output values are logic. Table II demonstrates the performance of applying different fusion levels and rules. As it can be observed from Table II, the average rule has equal EER values to the summation rule in all fusion methods. This is due to the variance levels of analysed information as these levels have the same proportional difference for both rules, which leads to the same probabilistic behaviour by the APNN. The multiplication rule has worst performance in all fusion methods except the ScLF. The reason for this is that the multiplication rule significantly affects the level of the data. This has caused increasing the differences between the learning and testing values in Equation (8) of the APNN. On the other hand, the multiplication rule played an effective role in the ScLF and this is not strange as increasing the variances in this level of the APNN produces distinct relationships between the values. Consequently, the final outputs of the APNN in the decision layer obtain advantages from this fusion. The results of the maximum and minimum rules vary between them according to their position in each fusion level. They have recorded equal EER values in the SLF because they generated small effective values from the low resolution FT images. The maximum rule has attained a noticeable performance in the FLF, while the minimum rule has recorded unsatisfied performance. This is due to the features of the FT images after the ELLBP operation, where the specifications are being clearer, and the maximum rule can maintain the textures. On the other hand, the minimum rule would select the least significant features. Both rules have comparable performance in the ScLF as their influences are nearly similar in this level. Again, there is an obvious difference between both rules in the DLF, because in this level the maximum rule selects the best obtained decisions between the combined samples. However, the minimum rule will collect the worst achieving decisions between the fused values. So, the maximum rule has reported better EER performance than the minimum rule. The concatenation rule has been found to attain the best performance in the SLF and FLF due to increasing the considered information of FTs. This rule is usually employed in the FLF and it has been cited that this method has the ability to achieve a significant performance [49] . Interestingly, this method has achieved acceptable EER in the SLF as the input data to the biometric system were doubled and increasing the input information will enhance the biometric system performance as confirmed in [29]. Nonetheless, this rule cannot be considered as a best choice between all the fusion levels, where it has benchmarked inferior EER value in the ScLF. This is because the number of the score 
values has been expanded and this significantly influences the decision of the APNN as it has to select the best solution between all the determined score values. It can be noticed that the AND and OR fusion rules have attained exactly the same results as in the minimum and maximum rules, respectively. This is due to the calculations of these methods, where the resulting output vector after the maximum rule is similar to the resulting output vector after the OR rule because both are going to consider the largest values between the fused vectors. However, the output vector after the minimum rule is similar to the output vector after the AND rule because both are going to consider the lowest values between the fused vectors. Overall, the FLF appears to have better performance in different fusion rules such as average, summation, maximum and concatenation. The SLF and ScLF can give useful outcomes by carefully considering the applied rule. Generally, the DLF can be considered as the lowest effective level as it is the last operation in the system and it depends on the last collected results from the previous operations. This is expected because the DLF has limited information to be considered compared with other fusion levels [40]. Finally, the concatenation rule of the FLF has been selected as the best method, because this is the only rule which has attained the best EER value in two fusion levels (SLF and FLF) and the FLF has recorded the best performance against the other fusion levels for various rules (average, summation, maximum and concatenation). Previous FT studies such as [13,30,31] has utilized 1 testing sample and 5 learning samples for each participant in the MSCASIA database. Obviously, one testing sample is too small comparing with the number of learning samples. Thus, additional comparisons are constructed by increasing the number of testing samples and decreasing the number of learning samples. Fig. 6 demonstrates the performance of applying different numbers of testing and learning samples for the same hand fingers.

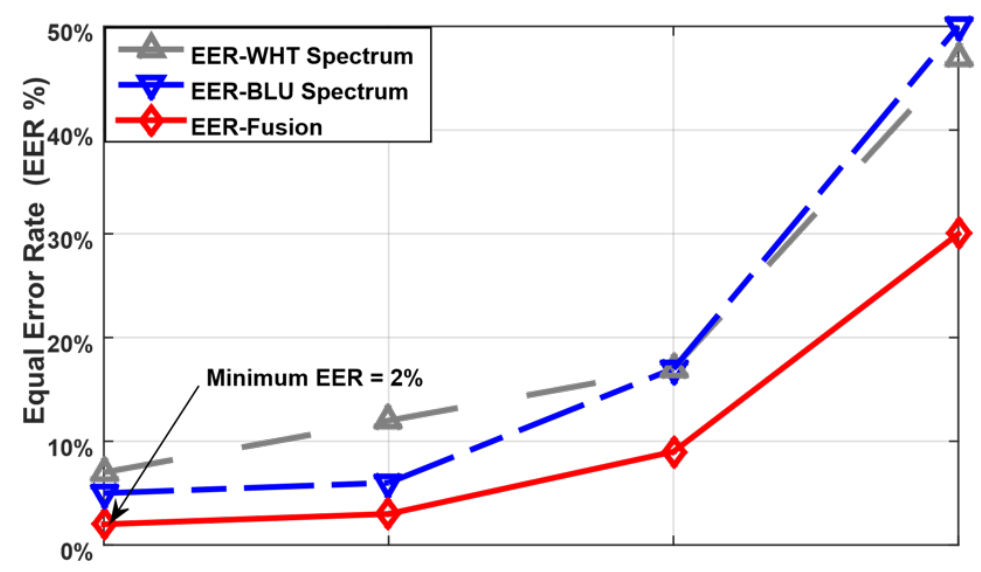

Figure 6: Comparisons of EER values for the WHT and BLU spectra by using different numbers of testing and learning samples of hand fingers

Figure 6 Comparisons of EER values for the WHT and BLU spectra by using different numbers of testing and learning samples of hand fingers. Figure 6 revealed that by reducing the number of learning samples, the EER percentages are reduced. That is, by using a high number of learning samples for the FTs of a single hand image, the best EER percentage has achieved 2\% after the combination or fusion. By including more number of testing samples like 2 and reducing the 
learning samples to 4 , this allows approximately $34 \%$ of the employed samples to be examined in the testing stage and around $66 \%$ of the employed samples to be utilized in the learning stage, a comparative EER percentage of $3 \%$ has been generated after the fusion. Now, by reducing the number of the learning samples further, the verification performance are dramatically reduced to $9 \%$ and $30 \%$ for the fusion after exploiting $50 \%$ and about $34 \%$ of the employed learning samples, respectively. Also, from the figure it can be observed that all the EER percentages have been enhanced after using the concatenation rule with the FLF comparing with the EER percentages of separately employing FTs of the WHT light and FTs of the BLU light.

\section{Conclusion}

This study presented different biometric systems by fusing between two different specifications of FTs. Acquiring finger images under different spectra can provide different FT specifications. Two types of spectra were considered. The first spectrum was for the normal WHT light and the second spectrum was for the BLU wavelength. Combining the FT specifications of the WHT and BLU spectra together can positively affect the performance of a human verification system. A large number of FT images from the MSCASIA was employed. That is, 4800 extracted FT images were used (2400 images from each spectrum). Four fusion levels were described and implemented in this work. These are the SLF, FLF, ScLF and DLF. All of these levels were examined under the following rules: average, summation, multiplication, maximum, minimum, concatenation (for the SLF, FLF and ScLF), AND and OR (for the DLF). The FLF with the concatenation rule has been chosen as the best method, because the concatenation rule has obtained a best EER value and the FLF has achieved remarkable performance. The suggested FT verification has attained EER percentages of 5\% and 7\% by separately employing the BLU and WHT lights. It is true that the combination system adds more stages and processes, however, the EER could be reduced to $2 \%$ after fusing the FT specifications for both types of lights.

\section{REFERENCES}

[1] R. Raid, "Human authentication with earprint for secure telephone system," Iraqi Journal of Computers, Communications, Control and Systems Engineering, vol. 12, no.2, pp.47 -56,2012.

[2] R. Raghavendra and C. Busch, "Novel image fusion scheme based on dependency measure for robust multispectral palmprint recognition," Pattern recognition, vol. 47, no. 6, pp.2205 -2221, 2014.

[3] S.-H. Lin, S.-Y. Kung, and L.-J. Lin, "Face recognition/detection by probabilistic decisionbased neural network," IEEE Transactions on Neural Networks, vol. 8, no. 1, pp. $114-132,1997$.

[4] F. S. Mustafa and R. R. Al-Nima, "Face recognition using invariant moments features," Tikrit Journal of Pure Science, vol. 14, no. 2, pp. 253 -259, 2009.

[5] Z. Zhou, E. Du, N. Thomas, and E. Delp, "A new human identification method: Sclera recognition," IEEE Transactions on Systems, Man and Cybernetics, Part A: Systems and Humans, vol. 42, no. 3, pp. $571-583,2012$.

[6] M. R. Khalil, M. S. Majeed, and R. R. Omar, "Personal identification with iris patterns," in 1st Scientific Conference for Information Technology, Dec 2008.

[7] "Personal identification with iris patterns," AL-Rafidain Journal of Computer Sciences and Mathematics, vol. 6, no. 1, pp. 13 -26, 2009.

[8] M. T. S. Al-Kaltakchi, W. L. Woo, S. S. Dlay, and J. A. Chambers, "Study of statistical robust closed set speaker identification with feature and score-based fusion," in IEEE Statistical Signal Processing Workshop (SSP), June 2016, pp. 1 -5.

[9] M. T. Al-Kaltakchi, W. L. Woo, S. Dlay, and J. A. Chambers, "Evaluation of a speaker identification system with and without fusion using three databases in the presence of noise and handset effects," EURASIP Journal on Advances in Signal Processing, vol. 2017, no. 1, p. 80, 2017.

[10] M. T. Al-Kaltakchi, W. L. Woo, S. S. Dlay, and J. A. Chambers, "Speaker identification evaluation based on the speech biometric and i-vector model using the timit and ntimit databases," in 5th International Workshop on Biometrics and Forensics (IWBF). IEEE, 2017, pp. 1 -6.

[11] "Comparison of i-vector and gmm-ubm approaches to speaker identification with timit and nist 2008 databases in challenging environments," in 25th European Signal Processing Conference (EUSIPCO). IEEE, 2017, pp. $533-537$.

[12] R. R. Al-nima, "Design a biometric identification system based on the fusion of hand geometry and backhand patterns," Iraqi Journal of Statistical Science, vol. 10, no. 17, pp. $169-180,2010$. 
[13] R. R. Al-Nima, S. S. Dlay, W. L. Woo, and J. A. Chambers, "Efficient finger segmentation robust to hand alignment in imaging with application to human verification," in 5th IEEE International Workshop on Biometrics and Forensics (IWBF), 2017, pp. 1 -6.

[14] R. R. O. Al-Nima, "Signal processing and machine learning techniques for human verification based on finger textures," PhD thesis, School of Engineering, Newcastle University, 2017.

[15] P. Yu, D. Xu, and H. Zhou, "Feature level fusion using palmprint and finger geometry based on canonical correlation analysis," in 3rd International Conference on Advanced Computer Theory and Engineering (ICACTE), vol. 5, 2010, pp. V5 - 260 - V5 - 264.

[16] M. Ferrer, A. Morales, C. Travieso, and J. Alonso, "Low cost multimodal biometric identification system based on hand geometry, palm and finger print texture," in 41st Annual IEEE International Carnahan Conference on Security Technology, 2007, pp. 52 -58.

[17] S.Sagiro glu and N. Ozkaya, "An intelligent face features generation system from finger prints," Turkish Journal of Electrical Engineering \& Computer Sciences, vol. 17, no. 2, pp. 183 -203, 2009.

[18] R. R. Al-Nima, S. Dlay, and W. Woo, "A new approach to predicting physical biometrics from behavioural biometrics," World Academy of Science, Engineering and Technology, International Journal of Computer, Electrical, Automation, Control and Information Engineering, vol. 8, no. 11, pp. $1996-2001,2014$.

[19] B. Bhaskar and S. Veluchamy, "Hand based multibiometric authentication using local feature extraction," in International Conference on Recent Trends in Information Technology (ICRTIT), 2014, pp. $1-5$.

[20] R. Al-Nima, M. Al-Kaltakchi, S. Al-Sumaidaee, S. Dlay, W. Woo, T. Han, and J. Chambers, "Personal verification based on multi-spectral finger texture lighting images," IET Signal Processing, 2018.

[21] M. T. S. Al-Kaltakchi, W. L. Woo, S. S. Dlay, and J. A. Chambers, "Study of fusion strategies and exploiting the combination of MFCC and PNCC features for robust biometric speaker identification," in 4th International Conference on Biometrics and Forensics (IWBF), March 2016, pp. 1 -6.

[22] S. Ribaric and I. Fratric, "A biometric identification system based on eigenpalm and eigenfinger features," IEEE Transactions on Pattern Analysis and Machine Intelligence, vol. 27, no. 11, pp. 1698 -1709, 2005.

[23] N. Pavesic, S. Ribaric, and B. Grad, "Finger-based personal authentication: a comparison of feature-extraction methods based on principal component analysis, most discriminant features and regularised-direct linear discriminant analysis," IET Signal Processing, vol. 3, no. 4, pp. 269 -281, 2009.

[24] G. Michael, T. Connie, and A. Jin, "Robust palm print and knuckle print recognition system using a contactless approach," in 5th IEEE Conference on Industrial Electronics and Applications (ICIEA), 2010, pp. $323-329$.

[25] G. K. O. Michael, T. Connie, and A. T. B. Jin, "An innovative contactless palm print and knuckle print recognition system," Pattern Recognition Letters, vol. 31, no. 12, pp. $1708-1719,2010$.

[26] V. Kanhangad, A. Kumar, and D. Zhang, "A unified framework for contactless hand verification," IEEE Transactions on Information Forensics and Security, vol. 6, no. 3, pp. $1014-1027,2011$.

[27] A.-K. Kong and D. Zhang, "Competitive coding scheme for palmprint verification," in Proceedings of the 17th International Conference on Pattern Recognition (ICPR), vol. 1, 2004, pp. $520-523$ Vol.1.

[28] Y. Zhang, D. Sun, and Z. Qiu, "Hand-based single sample biometrics recognition," Neural Computing and Applications, vol. 21, no. 8, pp. $1835-1844,2012$.

[29] R. R. Al-Nima, S. S. Dlay, W. L. Woo, and J. A. Chambers, "Human authentication with finger textures based on image feature enhancement," in 2nd IET International Conference on Intelligent Signal Processing (ISP), 2015

[30] R. R. O. Al-Nima, S. S. Dlay, W. L. Woo, and J. A. Chambers, "A novel biometric approach to generate ROC curve from the probabilistic neural network," in 24th IEEE Signal Processing and Communication Application Conference (SIU), 2016, pp. 141 -144.

[31] R. R. O. Al-Nima, S. S. Dlay, S. A. M. Al-Sumaidaee, W. L. Woo, and J. A. Chambers, "Robust feature extraction and salvage schemes for finger texture based biometrics," IET Biometrics, vol. 6, no. 2, pp. 43 -52, 2017.

[32] R. Al-Nima, M. Abdullah, M. Al-Kaltakchi, S. Dlay, W. Woo, and J. Chambers, "Finger texture biometric verification exploiting multi-scale sobel angles local binary pattern features and score-based fusion," Elsevier, Digital Signal Processing, vol. 70, pp. 178 -189, 2017.

[33] M. A. M. Abdullah, S. S. Dlay, W. L. Woo, and J. A. Chambers, "Robust iris segmentation method based on a new active contour force with a noncircular normalization," IEEE Transactions on Systems, Man, and Cybernetics: Systems, vol. PP, no. 99, pp. $1-14,2016$.

[34] L. Junli, Y. Gengyun, and Z. Guanghui, "Evaluation of tobacco mixing uniformity based on chemical composition," in 31st Chinese Control Conference (CCC), 2012, pp. $7552-7555$.

[35] L. V. Fausett and P. Hall, Fundamentals of neural networks: architectures, algorithms, and applications. Prentice-Hall Englewood Cliffs, 1994.

[36] J. Kou, S. Xiong, S. Wan, and H. Liu, "The incremental probabilistic neural network," in 6th International Conference on Natural Computation (ICNC), vol. 3, 2010, pp. $1330-1333$.

[37] S. Shorrock, A. Yannopoulos, S. Dlay, and D. Atkinson, "Biometric verification of computer users with probabilistic and cascade forward neural networks," Advances in Physics, Electronics and Signal Processing Applications, pp. 267 -272, 2000.

[38] H. AlMahafzah, M. Imran, and H. Sheshadri, "Multibiometric: Feature level fusion using FKP multi-instance biometric," IJCSI International Journal of Computer Science Issues, vol. 9, no. 3, 2012.

[39] P. K. Atrey, M. A. Hossain, A. El Saddik, and M. S. Kankanhalli, "Multimodal fusion for multimedia analysis: a survey," Multimedia systems, vol. 16, no. 6, pp. $345-379,2010$.

[40] A. A. M. Al-Shiha, "Biometric face recognition using multilinear projection and artificial intelligence," PhD thesis, School of Electrical and Electronic Engineering, Newcastle University, 2013.

[41] "CASIA-MS-PalmprintV1." [Online]. Available: http://biometrics.idealtest.org/

[42] Z. Khan, A. Mian, and Y. Hu, "Contour code: Robust and efficient multispectral palmprint encoding for human recognition," in IEEE International Conference on Computer Vision (ICCV), 2011, pp. $1935-1942$.

[43] Z. Khan, F. Shafait, Y. Hu, and A. Mian, "Multispectral palmprint encoding and recognition," arXiv preprint arXiv:1402.2941, 2014.

[44] M. Heikkila, M. Pietik ainen, and C. Schmid, "description of interest regions with local binary patterns," Pattern recognition, vol. 42, no. 3, pp. $425-436,2009$.

[45] X. Fu and W. Wei, "Centralized binary patterns embedded with image euclidean distance for facial expression recognition," in Fourth International Conference on Natural Computation, 2008. ICNC'08., vol. 4. IEEE, 2008, pp. 115 -119. 
[46] Y. Tong, R. Chen, and Y. Cheng, 'Facial expression recognition algorithm using LGC based on horizontal and diagonal prior principle," OptikInternational Journal for Light and Electron Optics, vol. 125, no. 16, pp. 4186 -4189, 2014.

[47] L. Wolf, T. Hassner, and Y. Taigman, "Descriptor based methods in the wild," in Workshop on Faces in 'Real-Life' Images: Detection, Alignment, and Recognition, 2008.

[48] Q. Tao and R. Veldhuis, "Illumination normalization based on simplified local binary patterns for a face verification system," in Biometrics Symposium, 2007, pp. $1-6$.

[49] A. A. Ross and R. Govindarajan, 'Feature level fusion of hand and face biometrics," in Defense and Security. International Society for Optics and Photonics, 2005, pp. $196-204$. 